\title{
El beneficio del rastreo de deterioro cognitivo en adultos mayores resulta aún incierto
}

Benefit of cognitive impairment in older adults remains still uncertain

Lin JS. Ann Intern Med. 2013;159:601-612.

\section{Objetivos}

Evaluar sistemáticamente los beneficios, daños y precisión diagnóstica de los instrumentos breves de rastreo de deterioro cognitivo para adultos mayores residentes en la comunidad as como los beneficios y daños de las estrategias terapéuticas comúnmente utilizadas en los pacientes con deterioro cognitivo mínimo y demencia así como en sus cuidadores.

\section{Fuentes de datos}

MEDLINE, Cochrane Database of Systematics Reviews, Cochrane Central Register of Controlled Trials, Database of Abstracts of Reviews of Effects (DARE), Agency for Healthcare Research and Quality (AHRQ), National Institute for Health and Care Excellence (NICE) y PsycINFO, desde 2001.

\section{Selección de estudios}

Se seleccionaron artículos en inglés de calidad buena o aceptable habían evaluado instrumentos de rastreo e intervenciones dirigidos a individuos residentes en la comunidad con deterioro cognitivo mínimo o demencia leve a moderada. Dichos instrumentos debían ser de aplicación breve (10 minutos o menos cuando se realizaban por un médico, o 20 minutos o menos cuando se auto-administraban al paciente o a un tercero). Se excluyeron estudios que habían evaluado pacientes con demencia por enfermedad de Parkinson.

\section{Extracción de datos}

Fue realizada por dos revisores independientes. Se utilizaron criterios específicos de calidad normalizados por la USPSTF los cuales se suplementaron con listas de cotejo de calidad del
NICE y AMSTAR para revisiones sistemáticas, NewcastleOttawa para estudios observacionales y QUADAS para estudios de diagnóstico.

\section{Resultados principales}

Respondieron a las siguientes preguntas: 1) El rastreo de deterioro cognitivo en adultos mayores no institucionalizados ¿mejora resultados relacionados a toma de decisiones desde la perspectiva del paciente/familia/cuidadores/sociedad?; 2) ¿Cuáles son las características operativas de los instrumentos de rastreo utilizados para detectar deterioro cognitivo?; 3) ¿Cuáles son los daños asociados al rastreo de deterioro cognitivo?; 4) Las intervenciones sobre deterioro cognitivo mínimo y demencia leve a moderada ¿mejoran resultados relacionados a toma de decisiones desde la perspectiva del paciente/familia/cuidadores/sociedad?; 5) ¿Cuáles son los daños asociados al tratamiento del deterioro cognitivo?

No se encontraron ensayos clínicos que respondan a la primera ni la tercera pregunta. Sin embargo, en un estudio la mitad de los pacientes con rastreo positivo para deterioro cognitivo declinaron en continuar la evaluación formal.

Respecto del segundo interrogante, se identificaron 55 estudios que evaluaron la precisión diagnóstica de los instrumentos utilizados para el rastreo de deterioro cognitivo. La tabla 1 resume las características operativas de aquellos que fueron evaluados en al menos dos estudios. Los estándares de referencia diagnósticos más utilizados fueron los criterios del DSM III, DSM IV y NINCDS-ADRDA. Fueron detectados 22 instrumentos de rastreo para deterioro cognitivo mínimo (DCM), en general con menores valores de sensibilidad.

Tabla 1: Precisión diagnóstica de los instrumentos breves para rastreo de demencia

\begin{tabular}{|c|c|c|c|}
\hline Instrumento & Estudios (participantes) & Rango Sensibilidad (IC95\%) & Rango Especificidad (IC95\%) \\
\hline \multicolumn{4}{|l|}{ Muy breves (<5min) } \\
\hline CDT & $6(2.170)$ & 67 a 97,9 (39 a 100) & 69 a $94,2(54$ a 97,1$)$ \\
\hline Mini-Cog & $4(1.570)$ & 76 a 100 (54 a 100) & 54 a $85,2(43$ a 88,4$)$ \\
\hline MIS & $5(1.971)$ & 43 a 86 (24 a 96$)$ & 93 a 97 (56 a 100) \\
\hline MSQ or SPMSQ & $4(1.057)$ & 92,3 a $100(29$ a 100$)$ & 86,5 a $100(76$ a 100$)$ \\
\hline Fluencia verbal & $6(2.083)$ & 37 a 89,5 (19 a 100) & 43 a 97 (33 a 99$)$ \\
\hline \multicolumn{4}{|l|}{ Breves (6 a 10 min) } \\
\hline MMSE & $14(10.185)$ & $88,3(81,3$ a 92,9$)$ & $86,2(81,8$ a 89,7$)$ \\
\hline AMT & $4(824)$ & 42 a $100(16$ a 100$)$ & 83 a 95,4 (76 a 99) \\
\hline FCSRT & $2(734)$ & 86 a 100 (41 a 100) & 73 a 87,2 (56 a 96) \\
\hline 7MS & $2(553)$ & 66,7 a 100 (NR a 100) & 95,1 a $100(86,8$ a 100$)$ \\
\hline TICS & $2(677)$ & 74 a 88 (54 a 96) & 86 a 97 (81 a 91$)$ \\
\hline \multicolumn{4}{|c|}{ Auto administrados (<20 min) } \\
\hline IQCODE & $5(1.251)$ & 75 a 87,6 (41 a 100$)$ & 65 a 91,1 (59 a 100$)$ \\
\hline
\end{tabular}

S: sensibilidad; E: especificidad; IC95\%: intervalo de confianza 95\%; 7MS: 7-Minute Screen; AMT: Abbreviated Mental Test; CDT: Clock Drawing Test; FCSRT: Free and Cued Selective Reminding Test; IQCODE: Informant Questionnaire on Cognitive Decline in the Elderly; MIS: Memory Impairment Screen; MIS-T: Memory Impairment Screen by Telephone; MMSE: Mini-Mental State Examination; MSQ: Mental Status Questionnaire; SPMSQ: Short Portable Mental Status Questionnaire; TICS: Telephone Interview for Cognitive Status. NR: no reportado.

Respecto de la cuarta pregunta, la tabla 2 meta-analiza algunos de los resultado de 48 estudios hallados $(n=18.390)$ en los cuales los inhibidores de la acetil colinesterasa mejoran la función cognitiva en el corto plazo. Sin embargo la magnitud de mejoría en el puntaje de ADAS-cog no reviste importancia clínica. Efectos similares se observan en 10 estudios $(n=3.465)$ que evaluaron memantina. La mayor parte de esta evidencia proviene de pacientes con demencia tipo Alzheimer con seis meses de seguimiento. En un subgrupo de estudios se observó una modesta mejoría en la función global. La discontinuación debido a efectos adversos fue más frecuente para los inhibidores de la acetil-colinesterasa.

Algunos estudios evaluaron también la efectividad de otros medicamentos o suplementos dietarios (ej, aspirina a bajas dosis, HMG-Co A reductasa, AINE, esteroides gonadales, multivitamínicos) pero ninguno demostró algún beneficio cognitivo o funcional. 
Tabla 2: Efecto de intervenciones farmacológicas en pacientes con deterioro cognitivo mínimo y demencia.

\begin{tabular}{l|c|c|c|c}
\multirow{2}{*}{ Droga } & \multicolumn{2}{|c|}{ Cambio en el puntaje de ADAS-Cog } & \multicolumn{2}{c}{ Suspensión debido a efectos adversos } \\
\cline { 2 - 5 } & Diferencia en la media de cambio (IC95\%) & $\mathbf{I}^{\mathbf{2}}$ & RR (IC95\%) & $\mathbf{I}^{\mathbf{2}}$ \\
\hline Donepezilo & $-2,03(-2,68$ a $-1,38)$ & $67,6 \%$ & $1,79(1,50$ a 2,13$)$ & $10,3 \%$ \\
\hline Galantamina & $-2,25(-2,94$ a $-1,55)$ & $68,4 \%$ & $2,50(1,78$ a 3,50$)$ & $67,5 \%$ \\
\hline Rivastigmina & $-3,06(-4,48$ a $-1,65)$ & $92,6 \%$ & $2,35(1,71$ a 3,21$)$ & $44,6 \%$ \\
\hline Memantina & $-1,36(-2,02$ a $-0,70)$ & $31,5 \%$ & $1,03(0,77$ a 1,38$)$ & $35,2 \%$ \\
\hline
\end{tabular}

RR: riesgo relativo; IC95\%: intervalo de confianza 95\%; £Un cambio de cuatro o más puntos en el puntaje de ADAS-cog durante seis meses se considera una mejoría clínica significativa en la demencia leve a moderada.

La tabla 3 meta-analiza los hallazgos de 59 trabajos hallados $(n=8.932)$ sobre intervenciones hacia cuidadores y pacientes que determinaron al menos efectos beneficiosos leves en cuanto a reducir la sobrecarga y depresión de los cuidadores luego de implementar una variedad heterogénea de interven- ciones psicoeducativas y de soporte, y efecto moderados (pero con moderada heterogeneidad) al introducir estrategias de estimulación cognitiva en pacientes. Los efectos de intervenciones de tipo actividad física o multidisciplinarias a pacientes mostraron efectos inconsistentes.

Tabla 3: Efecto de diferentes intervenciones no farmacológicas sobre pacientes con deterioro cognitivo mínimo y demencia y sus cuidadores.

\begin{tabular}{l|c|c}
\multirow{2}{*}{\multicolumn{1}{c|}{ Tipo de intervención }} & \multicolumn{2}{c}{ Resultado } \\
\cline { 2 - 3 } & Hedge-g£ & $\mathrm{I}^{2}$ \\
\hline Psicoeducativa dirigida a cuidadores evaluando sobrecarga & $-0,23(-0,35 \mathrm{a}-0,12)$ & $52,7 \%$ \\
\hline Psicoeducativa dirigida a cuidadores evaluando depresión & $-0,21(-0,30 \mathrm{a}-0,13)$ & $34,1 \%$ \\
\hline Estimulación cognitiva dirigida a pacientes & $-0,59(-0,93$ a $-0,25)$ & $52,7 \%$ \\
\hline
\end{tabular}

£Hedge-g: Diferencia media estandarizada (valores de 0,2 a menos de 0,5 se consideran cambios pequeños, aquellos de 0,5 a menos de 0,8 se consideran intermedios, y aquellos de 0,8 o mayores se consideran importantes; los valores negativos favorecen a la intervención sobre los controles).

Fuente de financiamiento: Agency for Healthcare Research and Quality (AHRQ)

\section{Comentario}

La presente revisión ha servido de soporte para reafirmar la clasificación I de la USPSTF (evidencia insuficiente para evaluar el balance entre beneficios y riesgos) respecto del rastreo de deterioro cognitivo y demencia en adultos mayores emitida por última vez en $2003^{1}$. Esta recomendación se encuentra en línea con los postulados de otras organizaciones.

La práctica habitual en atención primaria está en general acorde a esta incertidumbre y prioriza la búsqueda y detección de pacientes con deterioro cognitivo ante la identificación por parte del médico o la manifestación, por parte del propio paciente o un familiar, de síntomas (en general olvidos o alteraciones en el desempeño funcional).

Si bien los médicos de atención primaria sabemos que existen relativamente escasos recursos para ofrecer que puedan enlentecer o detener el avance de este padecimiento, existe consenso (aunque no evidencia dura) en que el diagnóstico oportuno puede brindar las bases para reinterpretar algunos síntomas, establecer expectativas a futuro, maximizar cuestiones relacionadas con el ejercicio de la autonomía y toma de decisiones futuras, entre otras y así reorganizar el cuidado.

El interrogatorio semiestructurado, junto con la administración de pruebas sencillas y muy difundidas como lo son el MMSE y el test del reloj suelen servir para iniciar la evaluación ${ }^{2}$. Algunas variantes algo más extensas del MMSE han sido validadas en nuestro medio para identificar pacientes en estadios más incipientes de demencia ${ }^{3}$.

Vale la pena insistir en que hasta el momento la evidencia resulta insuficiente para recomendar a favor o en contra del rastreo del deterioro cognitivo. Sin embargo, al ser esta una entidad prevalente en los adultos mayores y que genera importante discapacidad, es fundamental estar alertas a sus primeras manifestaciones para planificar junto al paciente y su familia pautas de cuidado.

Gabriel Villalón [ Servicio de Medicina Familiar y Comunitaria del Hospital Italiano de Buenos Aires. gabriel.villalon@ hospitalitaliano.org.ar ]

Villalón G. El beneficio del rastreo de deterioro cognitivo en adultos mayores resulta aún incierto. Evid Act Pract Ambul. 2015:18(1).Ene-Mar.5. Comentado de: Lin JS, O'Connor E, Rossom E. Screening for Cognitive Impairment in Older Adults: A Systematic Review for the U.S. Preventive Services Task Force. Ann Intern Med. 2013;159:601-612. PMID: 24145578.

\section{Referencias}

1. Boustani M, Peterson B, Hanson L, Harris R, Lohr KN. Screening for dementia in primary care: a summary of the evidence for the U.S. Preventive Services Task Force. Ann Intern Med. 2003;138:927-37.

2. Queja cognitiva y demencia. En: PROFAM, Salud del Anciano. Rubinstein E, et al Editores. 3ra edición. Buenos Aires: Fundación MF "Para el desarrollo de la Medicina Familiar y la Atención primaria de la salud", 2006.

3. Villalón G. Diagnóstico de demencia leve en atención primaria. Evid actual práct Ambul; 12(2):66-71, Abril - Junio 2009. 